Int. J. Contemp. Math. Sciences, Vol. 2, 2007, no. 32, 1587 - 1593

\title{
Single Step Two Stage Method For Second Order Singular Initial Value Problems
}

\author{
H. R. Khatami \\ Department of Mathematics \\ Islamic Azad University of Karaj Branch, Karaj, Iran \\ J. Rashidinia \\ Department of Mathematics \\ Islamic Azad University of Karaj Branch, Karaj, Iran \\ rashidinia@iust.ac.ir
}

\begin{abstract}
The classical Runge-Kutta-Nystrom methods cannot be applied directly to solve second order singular initial value problem, due to the singularity at $x=0$, These methods require some special procedure to obtain the solution at the first step and are thus not self starting. We have obtained the self starting explicit two stage Runge-Kutta-Nystrom method .Presented method is exact for $y=\frac{1}{x}$ besides having the polynomial order. The numerical result for two problems obtained and compared with the classical Runge- Kutta-Nystrom method. The numerical results demonstrate their superiority over the classical method.
\end{abstract}

Mathematics Subject Classification: 65L10

Keywords: Two stage Runge-Kutta-Nystrom method, Classical two stage Runge-Kutta- Nystrom

\section{Introduction}

The application of explicit Runge-Kutta schemes to obtain the numerical solution of the singular initial value problem of the form

$$
\begin{aligned}
& \frac{d Y}{d x}-M \frac{Y}{x}=g(S, Y)+f(x, Y), \quad x>0, \\
& y(0)=\eta,
\end{aligned}
$$


where $Y \in C^{1}[0,1], Y, g$ and $f$ vector functions, $M, S$ are constant matrices and $\eta$ is a constant vector. The application of explicit Runge-Kutta methods to obtain the numerical solution of equation (1) has been studied by Hoog and Weiss [2]. It is shown that, in general, the order of convergence is at most two but that the usual order upto a logarithmic term can be obtained for three and four stage schemes when applied to speclfic problems. Earlier Keller and Wolfe [4], Parter Stem and Estein [5] and Rentrop [6] showed that the explicit.RungeKutta schemes may be used to get satisfactory starting values, for computing the numerical solutions, on the entire interval, for equations similar to (1). However, at times it is advantageous to treat the higher order initial value problem in the original form especially if they are of the second order. In this paper we consider the singular initial value problem with spherical symmetry.

$$
y^{\prime \prime}+\frac{2}{x} y^{\prime}=f(x, y), x>0, y(0)=A, y^{\prime}(0)=0
$$

The classical Runge-Kutta-Nystrom methods [1,3] can not be applied directly to solve (3) due to the singularity at $x=0$. These methods require some special procedure to obtain the solution at the first step and are thus not self starting. We have obtained the self starting explicit two stage Runge-KuttaNystrom method for solving (3). This method is exact for $\frac{1}{x}$ besides having the polynomial order. The numerical results for the classical Runge-KuttaNystrom method have been compared with the exact solution. The numerical results and the self starting nature of the present methods demonstrate their superiority over the classical methods.

\section{Derivation of Method}

We define the two-stage Runge-Kutta-Nystrom method for (3) in the form

$$
\begin{aligned}
y_{j+1} & =y_{j}+\left(\frac{x_{j}}{x_{j+1}}\right) h y_{j}^{\prime}+w_{1} x_{j} k_{1}+w_{2} x_{j+1} k_{2}, \\
y_{j+1}^{\prime} & =\left(\frac{x_{j}}{x_{j}+1}\right)^{2} y_{j}^{\prime}+\frac{1}{h}\left(w_{1}^{\prime} x_{j} k_{1}+w_{2}^{\prime} x_{j+1} k_{2}\right), \\
k_{1} & =h^{2} f\left(x_{j}, y_{j}\right), \\
k_{1} & =h^{2} f\left(x_{j}+h, \bar{y}_{j+1}\right),
\end{aligned}
$$

and

$$
\bar{y}_{j+1}=y_{j}+\frac{x_{j}}{x_{j+1}} h \bar{y}_{j}+\beta_{21} k_{1},
$$




$$
\begin{aligned}
& =y_{j}+\frac{x_{j}}{x_{j+1}} h y_{j}^{\prime}+h^{2}+h^{2} \beta_{21}\left(y_{j}^{\prime \prime}+\frac{2}{x_{j}} y_{j}^{\prime}\right), \\
& =y_{j}+\left(\frac{x_{j}}{x_{j+1}}+\frac{2 h}{x_{j}} \beta_{21}\right) h y_{j}^{\prime}+h^{2} \beta_{21} y_{j}^{\prime \prime},
\end{aligned}
$$

$y_{j+1}$ is an approximation to the exact value $y\left(x_{j+1}\right)$. The method (4) is exact for $y=1$ and $\frac{1}{x}$ for any choice of the parameters $w_{j}, w_{j}^{\prime}$ and $\beta_{21}$, such that the method (4) is of a particular polynomial order.

We also have

$y\left(x_{j+1}\right)=y_{j}+h y_{j}^{\prime}+\frac{1}{2} h^{2} y_{j}^{\prime \prime}+\frac{1}{6} h^{3} y_{j}^{\prime \prime \prime}+\ldots$

Subtracting (7) from (6) we get

$\bar{y}_{j+1}=y\left(x_{j+1}\right)+\left(\frac{x_{j}}{x_{j+1}}+\frac{2 h}{x_{j}} \beta_{21}-1\right) h y_{j}^{\prime}+\left(\beta_{21}-\frac{1}{2}\right) h^{2} y_{j}^{\prime \prime}-\frac{h^{3}}{6} y_{j}^{\prime \prime \prime}+\ldots$

To make $\bar{y}_{j}$ agree with $y\left(x_{j+1}\right)$ to as high an order derivative as possible, we choose $\beta_{21}$, such that the coefficient of $y_{j}^{\prime}$ in (8) becomes zero. Thus we get.

$$
\frac{x_{j}}{x_{j+1}}+\frac{2 h}{x_{j}} \beta_{21}-1=0
$$

which gives

$\beta_{21}=\frac{1}{2} \frac{x_{j}}{x_{j+1}}$,

and

$$
\bar{y}_{j+1}=y\left(x_{j+1}\right)-\frac{h^{3}}{6}\left(y_{j}^{\prime \prime \prime}+\frac{3}{x_{j+1}} y_{j}^{\prime \prime}\right)+\ldots
$$

using (10) in (5-b), we find

$$
\begin{aligned}
k_{2} & =h^{2} f\left(x_{j+1}, y\left(x_{j+1}\right)-\frac{h^{3}}{6}\left(y_{j}^{\prime \prime \prime}+\frac{3}{x_{j+1}} y_{j}^{\prime \prime}\right)+O\left(h^{4}\right)\right) \\
& =h^{2}\left[f_{j+1}-\frac{h^{3}}{6}\left(y_{j}^{\prime \prime \prime}+\frac{3}{x_{j+1}} y_{j}^{\prime \prime}\right)\left(\frac{\partial f}{\partial y}\right) x_{j+1}+O\left(h^{4}\right)\right],
\end{aligned}
$$

using (5-a) and (11)we get from (4)

$$
\begin{aligned}
y_{j+1}= & y_{j}+\frac{x_{j}}{x_{j+1}} h y_{j}^{\prime}+h^{2}\left(W_{1}\left(x_{j} y_{j}^{\prime \prime}+2 y_{j}^{\prime}\right)+W_{2}\left(x_{j+1}+y_{j+1}^{\prime \prime}+2 y_{j+1}^{\prime}\right)\right) \\
& -\frac{h^{3}}{6} W_{2} x_{j+1}\left(y_{j}^{\prime \prime \prime}+\frac{3}{x_{j+1}} y_{j}^{\prime \prime}\right)\left(\frac{\partial f}{\partial y}\right) x_{j+1}+O\left(h^{4}\right) .
\end{aligned}
$$


Expanding both sides of (12) in Taylor's series about the point $x_{j}$ and simplifying we obtain

$$
\begin{aligned}
& {\left[\left(\frac{x_{j}}{x_{j+1}}\right)+2 h\left(W_{1}+W_{2}\right)-1\right] h y_{j}^{\prime}+\left[x_{j} W_{1}+\left(x_{j+1}+2 h\right) W_{2}-\frac{1}{2}\right] h^{2} y_{j}^{\prime \prime}+} \\
& {\left[\left(x_{j+1}+h\right) W_{2}-\frac{1}{6}\right] h^{3} y_{j}^{\prime \prime \prime}+\left[\frac{1}{2}\left(x_{j+1}+\frac{1}{3} h\right) W_{2}-\frac{1}{24}\right] h^{4} y_{j}^{(4)}+\ldots=0 .}
\end{aligned}
$$

Equating the coefficients of $y_{j}^{\prime}$ and $y_{j}^{\prime \prime}$ in (13) to zero, we obtain the equations

$$
\begin{aligned}
& \frac{x}{x_{j+1}}+2 h\left(W_{1}+W_{2}\right)=1, \\
& x_{j} W_{1}+\left(x_{j+1}+2 h\right) W_{2}=\frac{1}{2},
\end{aligned}
$$

which give

$W_{1}=\frac{2}{6 x_{j+1}}, \quad W_{2}=\frac{1}{6 x_{j+1}}$,

The first nonzero term in (13), which is the principal truncation error term in $y$ is given by

$$
\begin{aligned}
T_{y} & =\left[\frac{1}{6}-\left(x_{j+1}+h\right) W_{2}\right] h^{3} y_{j}^{\prime \prime \prime}=\left[\frac{1}{24}-\frac{1}{2} x_{j+1} W_{2}\right] h^{4} y_{j}^{(4)}+O\left(h^{5}\right) \\
& =-\frac{h^{4}}{24}\left[y_{j}^{(4)}+\frac{4}{x_{j+1}} y_{j}^{\prime \prime \prime}\right]+O\left(h^{5}\right),
\end{aligned}
$$

similarly using (5-a) and (11), we get from (4-b)

$$
\begin{aligned}
& y_{j+1}^{\prime}=\left(\frac{x_{j}}{x_{j+1}}\right)^{2} y_{j}^{\prime}+h\left[W_{1}^{\prime}\left(x_{j} y_{j}^{\prime \prime}+2 y_{j}^{\prime}\right)+W_{2}^{\prime}\left(x_{j+1} y_{j+1}^{\prime \prime}+2 y_{j+1}^{\prime}\right)\right] \\
& -\frac{1}{6} W_{2}^{\prime} x_{j+1}\left(y_{j}^{\prime \prime \prime}+\frac{3}{x_{j+1}} y_{j}^{\prime \prime}\right) h^{4}\left(\frac{\partial f}{\partial y}\right) x_{j+1}+O\left(h^{5}\right),
\end{aligned}
$$

Expanding both sides of (16) in Taylor series about the point $x_{j}$, collecting terms on one side and equation the coefficient of $y_{j}^{\prime}$ and $y_{j}^{\prime \prime}$ to zero, we obtain

$$
W_{1}^{\prime}=\frac{2 x_{j}+x_{j+1}}{6 x_{j+1}^{2}}, W_{2}^{\prime}=\frac{x_{j}+2 x_{j+1}}{6 x_{j+1}^{2}} .
$$


The principal truncation error term in $y^{\prime}$ reduces to

$T_{j}=-\frac{h^{3}}{12}\left(y_{j}^{4}+\frac{4}{x_{j+1}} y_{j}^{\prime \prime \prime}\right)+O\left(h^{4}\right)$.

Using the values of $W_{i}, W_{i}^{\prime}$ and $\beta_{21}$ in(4) we obtain the two stage method

$$
\begin{aligned}
y_{j+1} & =y_{j}+\left(\frac{x_{j}}{x_{j+1}}\right) h y_{j}^{\prime}+\frac{1}{6 x_{j+1}}\left[2 x_{j} K_{1}+x_{j+1} K_{2}\right], \\
y_{j+1}^{\prime} & =\left(\frac{x_{j}}{x_{j+1}}\right)^{2} y_{j}^{\prime}+\frac{1}{6 h x_{j+1}^{2}}\left[x_{j}\left(2 x_{j}+x_{j+1}\right) K_{1}+x_{j+1}\left(x_{j}+2 x_{j+1}\right) K_{2}\right], \\
K_{1} & =h^{2} f\left(x_{j}, y_{j}\right), \\
K_{2} & =h^{2} f\left(x_{j}+h, y_{j}+\frac{x_{j}}{x_{j+1}} h y_{j}^{\prime}+\frac{1}{2} \frac{x_{j}}{x_{j+1}} K_{1}\right),
\end{aligned}
$$

The error in $y$ end $y^{\prime}$ are given by (15) end (18).

The method (19) is exact for $y=\frac{1}{x}$ end has the polynomial order two.

\section{$3 \quad$ Numerical Results}

We have solved the following two problems

Problem 1:

$$
\begin{aligned}
& y^{\prime \prime}+\frac{2}{x} y^{\prime}=\lambda x^{\lambda-2}\left[1+\lambda+\lambda x^{\lambda}\right] y \\
& y(0)=1, \quad y^{\prime}(0)=0, \quad \lambda=2.5, \quad 0<x \leq 1 \\
& y(x)=\exp \left(x^{\lambda}\right)
\end{aligned}
$$

Problem 2:

$$
\begin{aligned}
& y^{\prime \prime}+\frac{2}{x} y^{\prime}=3 \cos x-x \sin x \\
& y(0)=1, \quad y^{\prime}(0)=0, \quad 0<x \leq 5 \\
& y(x)=x \sin x+\cos x
\end{aligned}
$$

By Using our modified two stage method (19) and classical two stage method [3 page 76] with the step size $h=\frac{1}{8}, \frac{1}{16}$ and $\frac{1}{32}$. It is observed that the maximum absolute error : $\left[\max \left|y_{j}-y\left(x_{j}\right)\right|\right]$ increases initially up to a certain value of $x$ and then starts decaying for all problems. The results are tabulated in tables 1 and 2 . 
Table 1: The maximum absolute errors of problem 1.

\begin{tabular}{ccc}
\hline$h$ & Modified method & Classical method \\
\hline$\frac{1}{8}$ & $6.244(-2)$ & $2.644(-2)$ \\
$\frac{1}{16}$ & $1.723(-3)$ & $4.559(-3)$ \\
$\frac{1}{32}$ & $4.551(-4)$ & $9.085(-4)$ \\
\multicolumn{1}{c}{ Error $y^{\prime}(x)$ Problem 1} \\
$\frac{1}{8}$ & $2.660(-1)$ & $1.111(-1)$ \\
$\frac{1}{16}$ & $7.769(-3)$ & $3.910(-2)$ \\
$\frac{1}{32}$ & $2.087(-3)$ & $1.380(-2)$ \\
\hline
\end{tabular}

Table 2: The maximum absolute errors of problem 2.

\begin{tabular}{ccc}
\hline$h$ & Modified method & Classical method \\
\hline$\frac{1}{8}$ & $8.990(-3)$ & $1.110(-2)$ \\
$\frac{1}{16}$ & $2.250(-4)$ & $2.719(-3)$ \\
$\frac{1}{32}$ & $5.626(-5)$ & $6.670(-4)$ \\
& Error $y^{\prime}(x)$ Problem 2 \\
$\frac{1}{8}$ & $7.348(-3)$ & $5.790(-3)$ \\
$\frac{1}{16}$ & $1.837(-4)$ & $1.374(-3)$ \\
$\frac{1}{32}$ & $4.595(-5)$ & $3.343(-4)$ \\
\hline
\end{tabular}

\section{Conclusion}

Self starting explicit two stage Runge-Kutta-Nystrom method for solving the singular initial value problems with spherical symmetry has been derived.

The classical Runge-Kutta-Nystrom method is not self starting. The numerical results and self starting nature of the present method demonstrate their superiority over the classical method.

ACKNOWLEDGEMENTS. This research is supported by Islamic Azad University of Karaj branch, Karaj- Iran.

\section{References}

[1] L. Collatz, The numerical treatment of differential equations, Springer-verlag, Berlin, 1966

[2] De. F. Hoog and R. Weiss, The application of Runge-Kutta schemes to singular initial value problems, Math's. Comput. 44 (1985), 93-103. 
[3] M. K. Jain, Numerical solution of differential equations, Wiley Eastern (2 $2^{\text {nd }}$ Ed.) 1984.

[4] H. B. Keller and A. W. Wolfe, On the non-unique equilibrium states and buckling mechanism of spherical shells, SIAM. J. 13 (1965), 674-705.

[5] S. V. Parter, M. L. Stein and P. R. Stein, on the multiplicity of solution of a differential equation arising in chemical reactor theory, Comput. Sc. Technical Report 194, Unv, of Wisconsin-Madison(1973)

[6] P. Rantrop, A Taylor series method for the numerical solution of two-point boundary value problems, Numer. Math. 31(1979), 359-375.

Received: February 11, 2007 